\title{
An Open-Source Hybrid CFD-DSMC Solver for High Speed Flows
}

\author{
D. E. R. Espinoza ${ }^{1,2, a)}$, V. Casseau ${ }^{1,2}$, T. J. Scanlon ${ }^{1,2}$ and R. E. Brown ${ }^{2}$ \\ ${ }^{1}$ James Weir Fluids Laboratory, Department of Mechanical and Aerospace Engineering, University of Strathclyde, \\ Glasgow, G1 1XJ, UK \\ ${ }^{2}$ Centre for Future Air-Space Transportation Technology, Department of Mechanical and Aerospace Engineering, \\ University of Strathclyde, Glasgow, GI IXJ, UK \\ ${ }^{a}$ Corresponding author: daniel.espinoza@ @strath.ac.uk
}

\begin{abstract}
During re-entry, a spacecraft will experience flow conditions ranging from highly rarefied to continuum. To simulate regions in between, a hydrodynamic-molecular gas hybrid solver should be used to provide accuracy and efficiency. Currently available hybrid codes are in-house codes or do not provide the capabilities to simulate all of the phenomena a spacecraft will experience during re-entry. An open-source CFD-DSMC hybrid code is being developed within the OpenFOAM framework, coupling the solvers dsmcFoam and hy2Foam. In this paper, comparison between the CFD, DSMC and hybrid codes have been performed for simple cases. The $d s m c F o a m$ and the hybrid code have shown to compare satisfactorily.
\end{abstract}

\section{INTRODUCTION}

During re-entry, spacecraft experience a wide range of flow conditions, from the highly-rarefied regime in the upper atmosphere down to the continuum regime at sea level. Whereas the simulation of the previously mentioned extreme conditions can be performed using the direct simulation Monte Carlo method (DSMC) and computational fluid dynamics (CFD) respectively, there are some regimes in between where the employment of only one of these techniques would be extremely computationally intense or physically inaccurate.

Numerous hybrid CFD-DSMC codes have been developed to tackle this problem. Most of these solvers have been reviewed by Schwartzentruber and Boyd [1], and are in-house codes. Open-source hybrids developed within the OpenFOAM framework [2] have also been proposed [3, 4], merging the compressible solver rhoCentralFoam $[5,6]$ and the particle-based DSMC solver $d s m c F o a m ~[7,8]$. dsmcFoam is suitable to accurately simulate re-entry flows, as it inherently has translational, rotational and vibrational energies, follows the quantum Larsen-Borgnakke internal energy distribution model [9,10], and uses the quantum-kinetic model [11] to describe the chemical reactions occurring in the flow. However, rhoCentralFoam, as a one-temperature solver without chemical modelling, lacks this capability. For this reason, the James Weir Fluids Laboratory at the University of Strathclyde is currently developing a two-temperature solver called hy2Foam $[12,13]$.

The purpose of this work is to couple the CFD solver hy2Foam and the DSMC solver dsmcFoam. Simple test cases have been simulated to compare the hybrid results to the pure DSMC results. In addition, different particle-generation distributions have been compared, and a study of how the particle type affects the final results has been performed.

\section{HYBRID SCHEME}

The most important aspects when dealing with a CFD-DSMC hybrid are how to determine the interface between the continuum and the particle regions, and how to transmit information between them [14]. 
Typically, the distinction between the regions is determined by applying a continuum breakdown parameter to the flow field. Quite a variety of parameters have been proposed to characterise the departure from the continuum regime [15-18], however, the present work defines a breakdown parameter using the gradient-length local Knudsen number[19], defined as:

$$
K n_{G L L-\phi}=\frac{\lambda}{\phi}|\nabla \phi|
$$

where $\lambda$ is the local mean free path and $\phi$ can be the gas density $\rho$, the gas translational-rotational or vibrational temperatures $T_{t r}, T_{v}$, or the velocity magnitude $|\mathbf{U}|$. In addition, the breakdown parameter should take into account the departure from thermal equilibrium [20], as this affects the hypothesis made by the continuum solver. This criterion can be defined as:

$$
f\left|\frac{T_{t}-T_{r}}{T_{r}}\right|
$$

where $f$ is a scaling factor, and $T_{t}$ and $T_{r}$ are the translational and rotational temperatures respectively. Aiming for $K n_{G L L}<0.05[14,19]$ and a departure from thermal equilibrium smaller than $1 \%$ [20], the flow is considered not to be continuum when:

$$
B r=\max \left(K n_{G L L-\rho}, K n_{G L L-T_{t r}}, K n_{G L L-T_{v}}, K n_{G L L-|\mathbf{U}|}, 5\left|\frac{T_{t}-T_{r}}{T_{r}}\right|\right)>0.05
$$

To transfer information between regions, there are two prevalent alternatives: flux-based coupling and state-based coupling. In flux-based coupling, mass, momentum and energy fluxes are calculated at the interface for both the CFD and DSMC regions. The DSMC flux is obtained by tracking the particles that cross the interface, whereas the CFD flux is obtained by extrapolating macroscopic gradients. These fluxes are in general different, so a modified flux would need to be calculated to ensure that the method is conservative. In addition, the statistical scatter for the flux from the DSMC region is greater than the one from state-based quantities (in fact the statistical error can be shown to be $E_{\text {flux }} \sim E_{\text {state }} / K n$ [21], and to reduce $E_{\text {flux }} \approx E_{\text {state }}$ the number of samples needed would scale as $1 / K n^{2}$ [14]). In state-based coupling [22, 23], each region extends some cells into the other, creating what it is called a buffer zone. In these cells, the macroscopic state from one solver is imposed onto the other. In this way, each solver will be responsible for its own fluxes at the interface, assuring conservation. DSMC particles in the CFD buffer region are used to obtain the macroscopic velocity, temperatures and densities that the CFD solver needs to resolve the flow, and the macroscopic fields obtained from CFD are used in the DSMC buffer cells to generate particles according to a suitable distribution.

Both coupling alternatives have been widely used in the development of hybrid codes ([24-29] for flux-based, $[3,14,20,30-32]$ for state-based). In this work, however, we will focus on state-based coupling as the information transfer method.

As the DSMC particles will be initialised using the CFD macroscopic fields as initial guesses, it is also important to take into account the distribution used to generate them. In this work, three possibilities have been considered.

As a first approximation, we can consider the flow to be in equilibrium. In this case, the thermal velocity follows a Maxwellian distribution function [33]:

$$
f^{(0)}(\mathbf{C})=\left(\frac{\beta^{3}}{\pi^{3 / 2}}\right) \exp (-\mathscr{C} \cdot \mathscr{C})
$$

where $\mathbf{C}=\mathbf{C}_{\mathbf{0}}-\mathbf{U}$ is the thermal velocity, $\mathbf{C}_{\mathbf{0}}$ is the particle velocity, $\mathbf{U}$ is the macroscopic velocity, $\beta=\sqrt{\frac{m}{2 k_{B} T_{t}}}$ is the inverse of the most probable thermal speed, $m$ is the mass of a particle, $k_{B}$ is the Boltzmann constant, and $\mathscr{C}=\beta \mathbf{C}$ is the scaled thermal velocity. This distribution is accurate when the CFD results are obtained using the Euler equations. If instead we consider the flow to be in a state of weak non-equilibrium, a more accurate approximation can be obtained using the Chapman-Enskog perturbation method on the Boltzmann equation. This approximation includes the effect of translational heat transfer and shear stress, and is an accurate representation of a Navier-Stokes-Fourier solution. The resulting distribution for the thermal velocity, called Chapman-Enskog distribution [34], can be represented as:

$$
\begin{aligned}
f^{(1)}(\mathbf{C}) & =f^{(0)}(\mathbf{C}) \Gamma(\mathbf{C}) \\
\Gamma(\mathbf{C}) & =1+\left(\mathbf{q}_{t} \cdot \mathscr{C}\right)\left[\frac{2}{5}(\mathscr{C} \cdot \mathscr{C})-1\right]-\mathscr{C} \cdot \tau \cdot \mathscr{C}
\end{aligned}
$$


where $\mathbf{q}_{t}=\frac{2 \beta}{p} \mathbf{q}_{t}^{N S}$ is the scaled translational heat flux, $p$ is the macroscopic pressure, $\tau=\frac{\tau^{\mathrm{NS}}}{p}$ is the scaled shear stress, and the superscript $N S$ represents the non-scaled quantity, according to the Navier-Stokes solution.

Chapman-Enskog distribution works well to represent the state of a single-species, monatomic flow. For multiple species, or molecules with internal degrees of freedom, the sources of non-equilibrium increase, as we have now to take into account the rotational and vibrational heat flux, as well as the species diffusion. For these flows, each species can be generated using the generalised Chapman-Enskog distribution [29, 35]. In this case, the equilibrium state is the Maxwell-Boltzmann distribution, defined for species $s$ as

$$
f_{s}^{(0)}\left(\mathbf{C}, \varepsilon_{r}, \varepsilon_{v}\right)=\left(\frac{\beta^{3}}{\pi^{3 / 2}}\right) \frac{g_{r} g_{v}}{Z_{r} Z_{v}} \exp \left[-(\mathscr{C} \cdot \mathscr{C})-\varepsilon_{r}-\varepsilon_{v}\right]
$$

where the subscripts $r, v$ represent rotational and vibrational properties respectively, $\varepsilon_{i}=\frac{\epsilon_{i}}{k_{B} T_{i}}$ is the scaled energy, $\epsilon_{i}$ is the energy, $g_{i}$ is the degeneracy, and $Z_{i}$ is the partition function. It can be assumed that the rotational relaxation occurs rapidly $[29,35]$, so $T_{t} \approx T_{r}$, and so for this work the scaled rotational energy will be defined as $\varepsilon_{r}=\frac{\epsilon_{r}}{k_{B} T_{t}}$. The generalised Chapman-Enskog distribution is defined as:

$$
\begin{aligned}
f_{s}^{(1)}\left(\mathbf{C}, \varepsilon_{r}, \varepsilon_{v}\right)= & f_{s}^{(0)}\left(\mathbf{C}, \varepsilon_{r}, \varepsilon_{v}\right) \Gamma_{s}\left(\mathbf{C}, \varepsilon_{r}, \varepsilon_{v}\right) \\
\Gamma_{s}\left(\mathbf{C}, \varepsilon_{r}, \varepsilon_{v}\right)= & 1+2\left(\mathscr{C} \cdot \mathscr{D}_{s}\right)+\left(\mathbf{q}_{t, s} \cdot \mathscr{C}\right)\left[\frac{2}{5}(\mathscr{C} \cdot \mathscr{C})-1\right]+\left(\mathbf{q}_{r, s} \cdot \mathscr{C}\right)\left(\varepsilon_{r}-\bar{\varepsilon}_{r, s}\right)+ \\
& +\left(\mathbf{q}_{v, s} \cdot \mathscr{C}\right)\left(\varepsilon_{v}-\bar{\varepsilon}_{v, s}\right)-\mathscr{C} \cdot \tau_{\mathbf{s}} \cdot \mathscr{C}
\end{aligned}
$$

where $\mathscr{D}=\frac{\beta}{\rho} \mathscr{D}^{N S}$ is the scaled diffusion flux, $\rho$ is the density, $\mathbf{q}_{i}=\frac{2 \beta}{\rho C_{v, i} T_{i}} \mathbf{q}_{i}^{N S}$ the scaled rotational $(i=r)$ or vibrational $(i=v)$ heat flux, $C_{v, i}$ the rotational or vibrational specific heat at constant volume, and $\bar{\varepsilon}_{i}$ the scaled average energy.

\section{RESULTS AND DISCUSSION}

To test the capabilities of the hybrid code, a heat transfer flow and a Couette flow have been simulated, for three different species, in increasing complexity: argon, modified nitrogen, and pure nitrogen. For all these simulations, the domain of the CFD and DSMC regions are the same, with a wall separation of $1 \mathrm{~m}$ and the DSMC region extending $14 \mathrm{~cm}$ from each wall. Figure 1 shows the domain, boundary conditions, and CFD and DSMC zones for these simulations. For each of the species, the density has been set such that the body-length Knudsen number $K n_{B L}$ is 0.05 . Studies on different breakdown parameters for similar test cases $[36,37]$ show that the non-equilibrium originates from the Knudsen layer near the wall. For this reason, the CFD and DSMC domains are consider appropriate for the complete simulation, and will not be updated.

\section{Argon}

Argon is a monatomic species, and thus lacks internal degrees of freedom. Simulations using argon should match single-temperature CFD models, so it should be the easiest case to simulate accurately. Figures 2 and 3 show the results for the heat transfer and Couette flow simulations respectively. It can be seen on that the temperature and velocity profiles match the DSMC results reasonably well. In Fig. 2b, a comparison for the translational temperature between the three possible distribution can be seen. As argon lacks internal degrees of freedom, the Chapman-Enskog and generalised Chapman-Enskog provide the same result. The Maxwellian distribution, however, is not sufficient to capture the non-equilibrium on the DSMC region. This error is then propagated to the CFD region during the coupling, leading to an incorrect solution.

\section{Vibrationless nitrogen}

Nitrogen is a diatomic molecule, thus having both rotational and vibrational energies. However, for this case the molecule has been artificially set to lack vibrational modes. The case should also match single-temperature CFD 


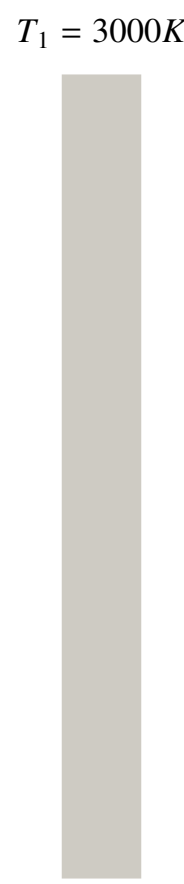

$$
T_{1}=3000 K ; U_{1}=300 \mathrm{~m} / \mathrm{s}
$$

$$
T_{0}=2000 K
$$

$$
T_{0}=2000 K ; U_{0}=0
$$

a) Heat transfer

$$
\text { b) Couette flow }
$$

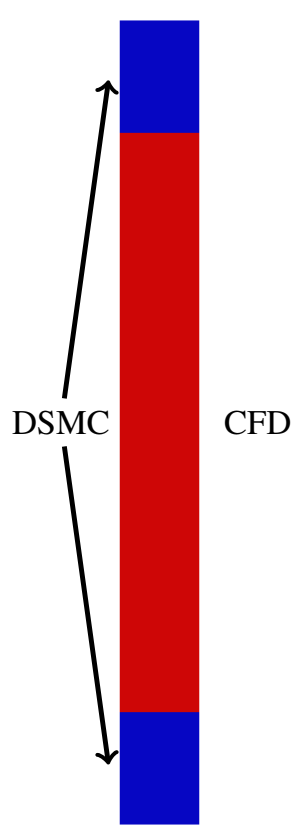

FIGURE 1. Boundary conditions and zones for the test cases

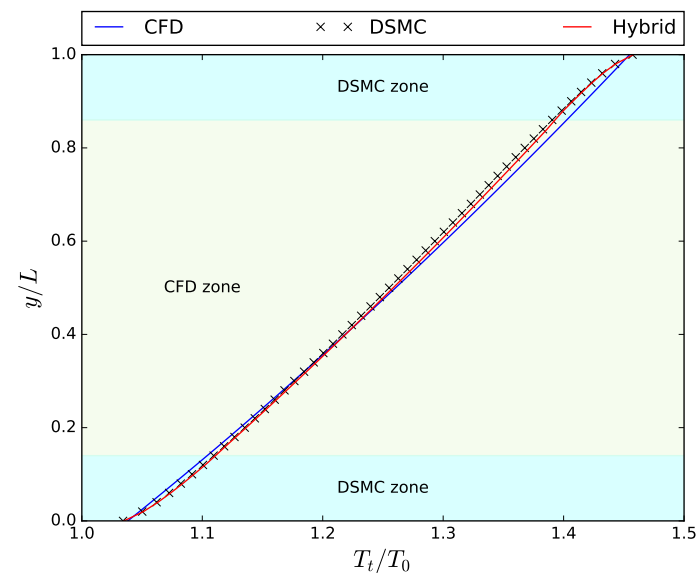

a) Translational temperature

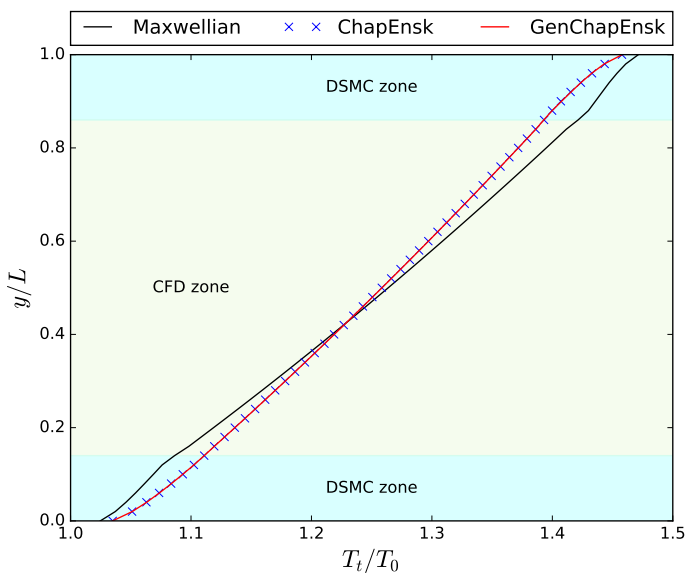

b) Distribution comparison

FIGURE 2. Results for the heat transfer simulation, for argon 


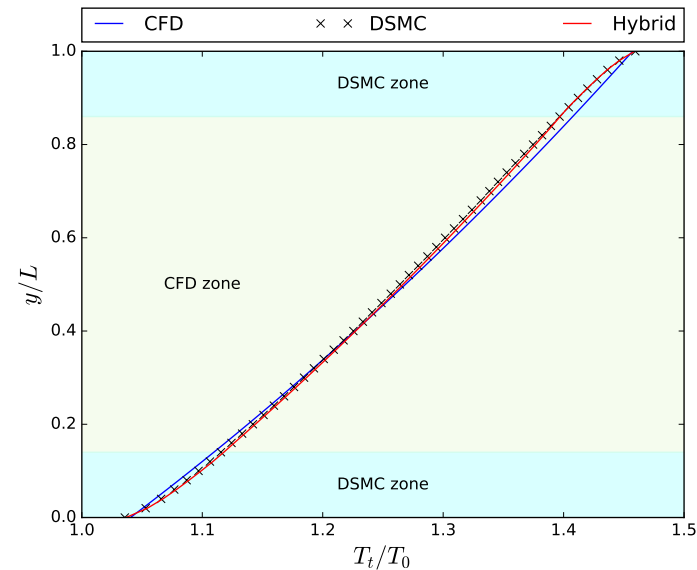

a) Translational temperature

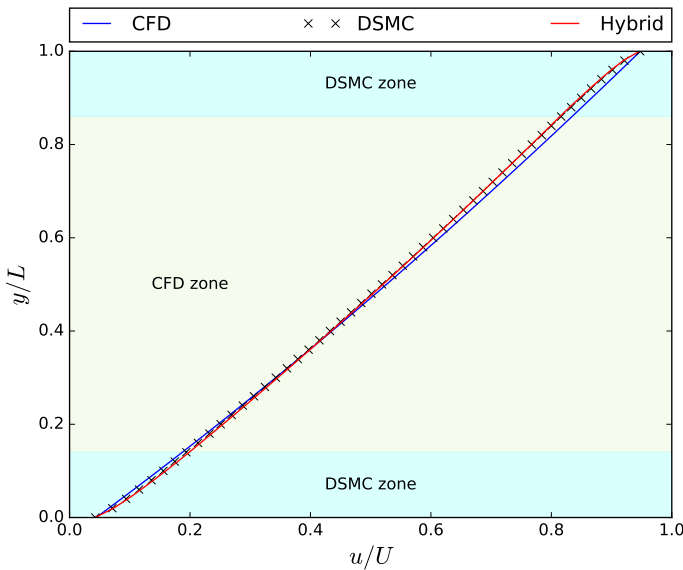

b) Velocity

FIGURE 3. Results for the Couette flow simulation, for argon

models, whereas DSMC should take into account the rotational energy as well as the translational. The hybrid code must be able to accurately capture this effect. Figures 4 and 5 show the results from the different simulations. As expected, the hybrid results for temperature and velocity match the DSMC results with high accuracy. Figure $4 \mathrm{~b}$ shows that even the Chapman-Enskog distribution is not enough for a flow with moderately high Knudsen number when we include the rotational energy.

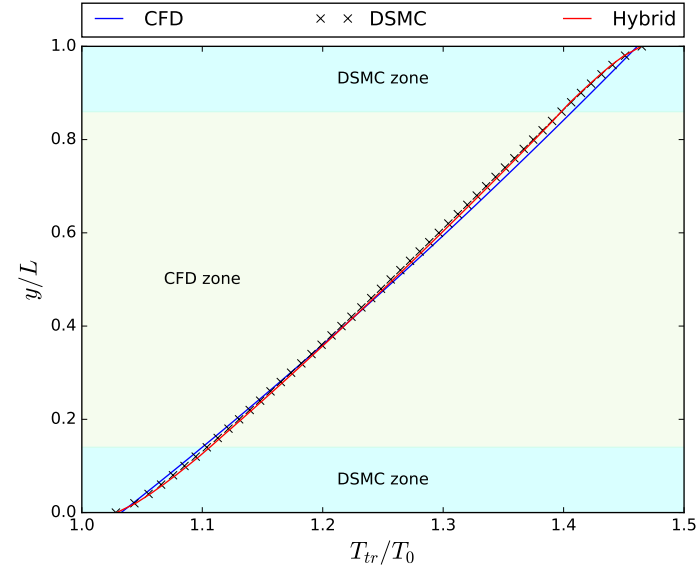

a) Transrotational temperature

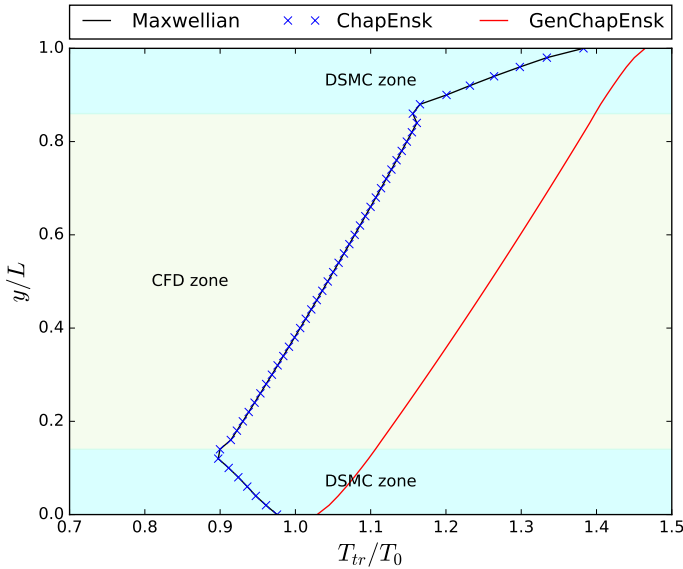

b) Distribution comparison

FIGURE 4. Results for the heat transfer simulation, for vibrationless Nitrogen

\section{Nitrogen}

Finally, a nitrogen molecule with all its degrees of freedom is simulated. Figures 6 to 9 show the results of the simulations. It can be seen that the hybrid can accurately solve flows that include all three temperatures. Figure 7 show the discrepancy between the distributions. As expected, for a molecule with internal energy, only the generalised Chapman-Enskog distribution provides enough accuracy. 


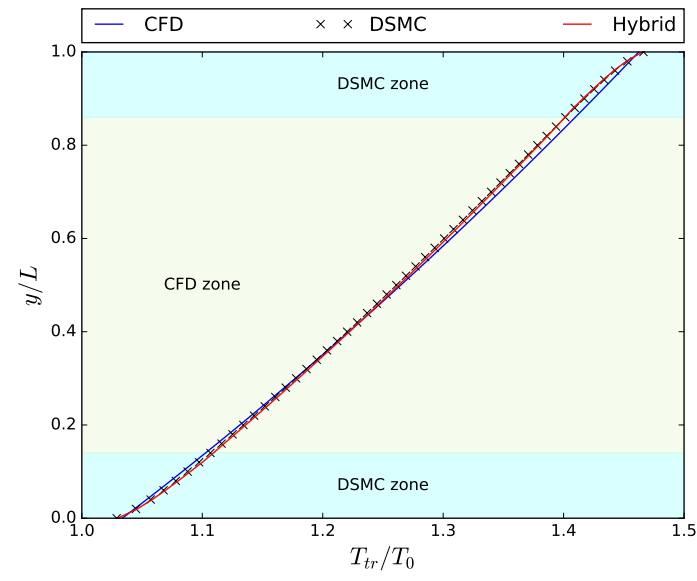

a) Transrotational temperature

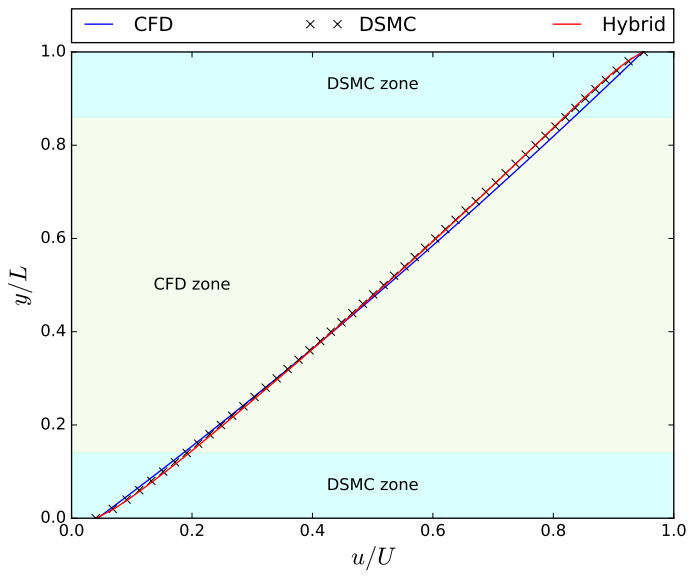

b) Velocity

FIGURE 5. Results for the Couette flow simulation, for vibrationless nitrogen

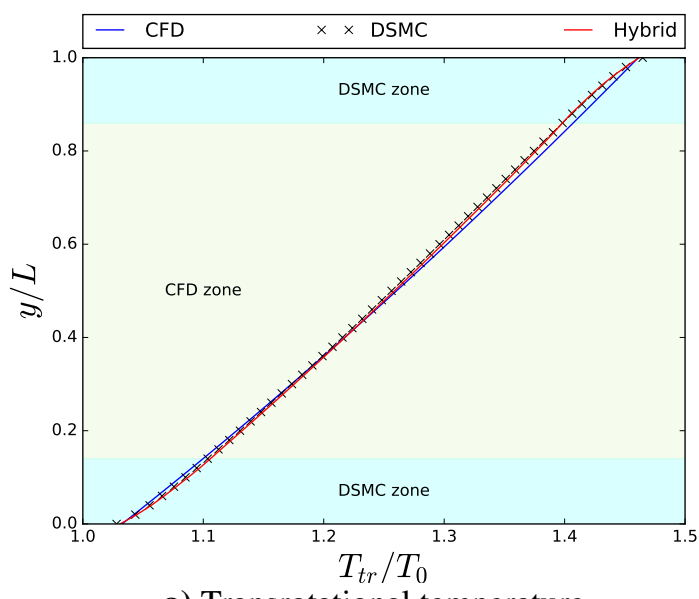

a) Transrotational temperature

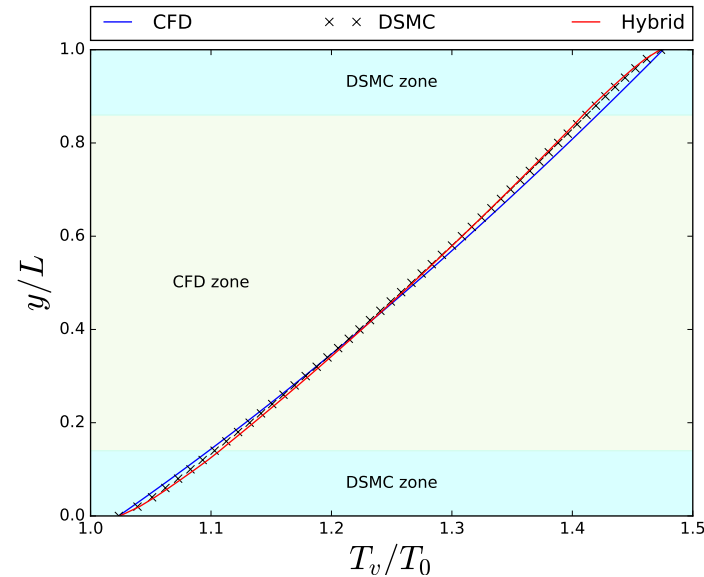

b) Vibrational temperature

FIGURE 6. Temperature results for the heat transfer simulation, for nitrogen

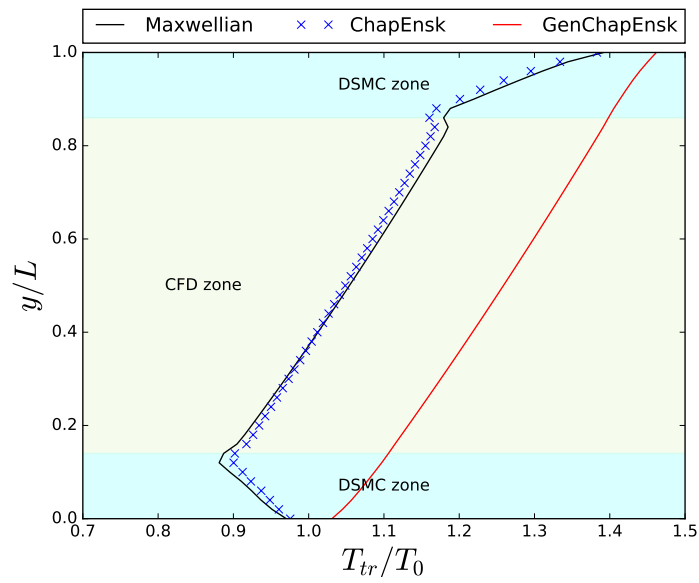

a) Distribution comparison, $T_{t r}$

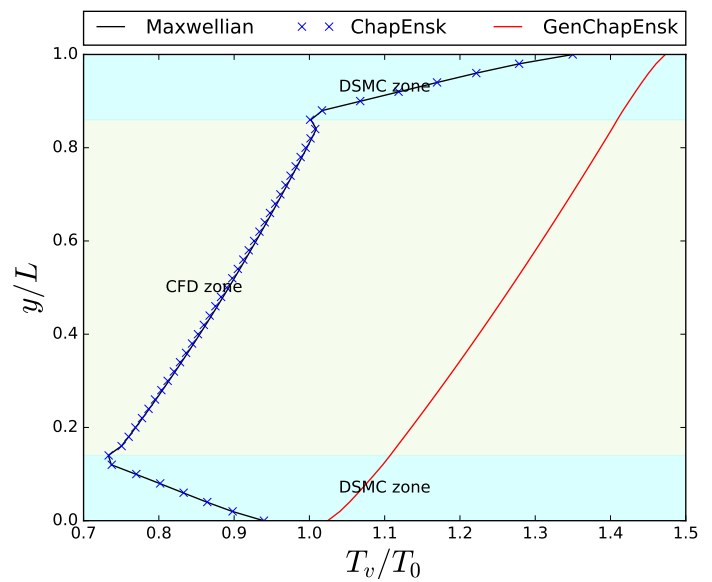

b) Distribution comparison, $T_{v}$

FIGURE 7. Distribution comparison for the heat transfer simulation, for nitrogen 


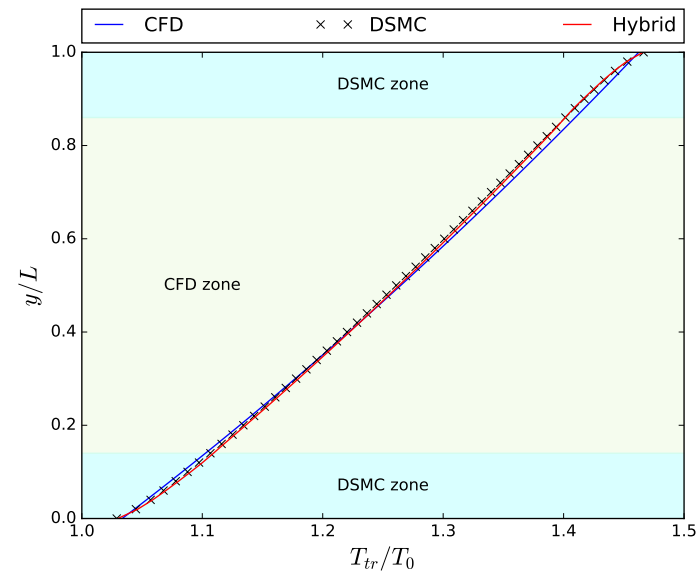

a) Transrotational temperature

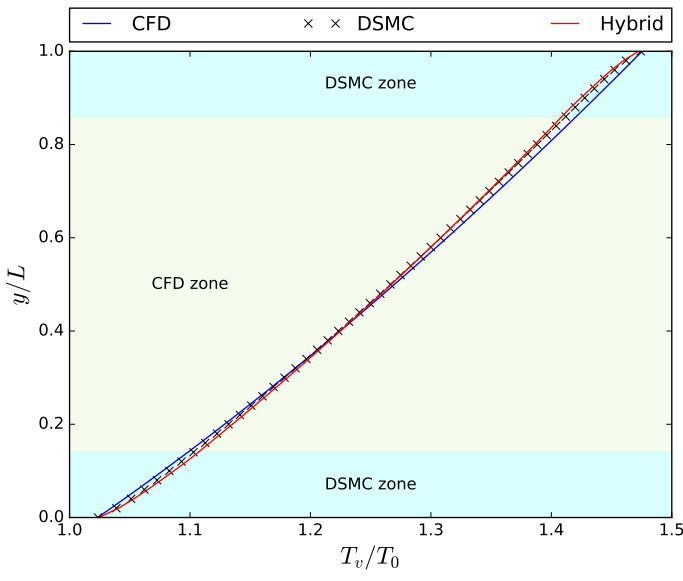

b) Vibrational temperature

FIGURE 8. Temperature results for the Couette flow simulation, for nitrogen

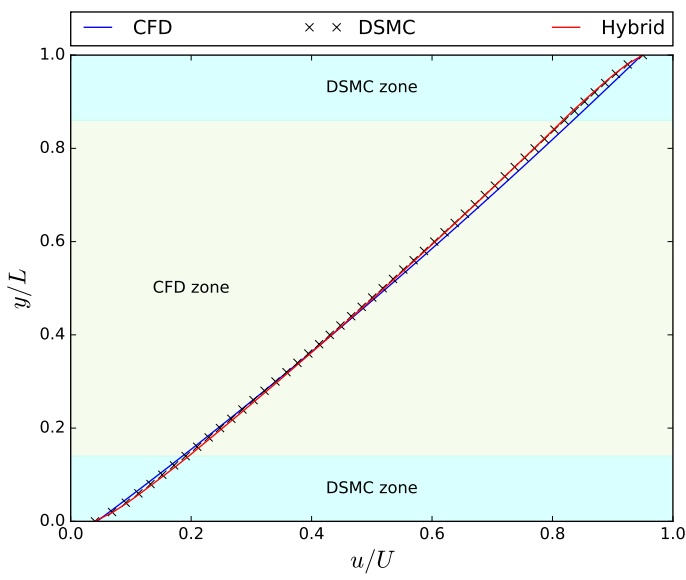

FIGURE 9. Velocity results for the Couette flow simulation, for nitrogen

\section{CONCLUSIONS}

The hybrid solver has been shown to approach the DSMC solution with high accuracy. The generalised ChapmanEnskog distribution has been proven to be the one that provides accurate results for all of these cases.

Future work will focus on extending the test cases to more complex simulations, as well as including multiple species.

\section{REFERENCES}

[1] T. E. Schwartzentruber and I. D. Boyd, Progress in Aerospace Sciences 72, 66-79 (2014).

[2] OpenFOAM official website, http://www.openfoam.org/ ( accessed: 15 June 2016).

[3] M. Darbandi and E. Roohi, International Journal for Numerical Methods in Fluids 72, 937-966 (2013).

[4] K. Gott, "A Hybrid CFD-DSMC Model Designed to Simulate Rapidly Rarefying Flow Fields and Its Application To Physical Vapor Deposition," Ph.D. thesis, The Pennsylvania State University 2015.

[5] C. J. Greenshields, H. G. Weller, L. Gasparini, and J. M. Reese, International Journal for Numerical Methods in Fluids 63, 1-21 (2010).

[6] L. F. G. Marcantoni, J. P. Tamagno, and S. A. Elaskar, Mecánica Computacional 31, 2939-2959 (2012). 
[7] T. J. Scanlon, E. Roohi, C. White, M. Darbandi, and J. M. Reese, Computers \& Fluids 39, 2078-2089 (2010).

[8] T. J. Scanlon, C. White, M. K. Borg, R. C. Palharini, E. Farbar, I. D. Boyd, J. M. Reese, and R. E. Brown, AIAA Journal 53, 1670-1680 (2015).

[9] C. Borgnakke and P. S. Larsen, Journal of Computational Physics 18, 405-420 (1975).

[10] F. Bergemann and I. D. Boyd, Progress in Astronautics and Aeronautics 158, p. 174 (1994).

[11] G. A. Bird, Physics of Fluids 23 (2011).

[12] V. Casseau, T. J. Scanlon, and R. E. Brown, "Development of a Two-Temperature Open-Source CFD Model for Hypersonic Reacting Flows," in 20th AIAA International Space Planes and Hypersonic Systems and Technologies Conference, Paper Number 3637 (Glasgow, UK, 2015).

[13] V. Casseau, T. J. Scanlon, B. John, D. R. Emerson, and R. E. Brown, "Hypersonic Simulations using OpenSource CFD and DSMC Solvers," in 30th International Symposium on Rarefied Gas Dynamics, Paper Number 2828 (Victoria, Canada, 2016).

[14] T. E. Schwartzentruber and I. D. Boyd, Journal of Computational Physics 215, 402-416 (2006).

[15] G. A. Bird, AIAA Journal 8, 1998-2003 (1970).

[16] S. Tiwari, Journal of Computational Physics 144, 710-726 (1998).

[17] A. L. Garcia and B. J. Alder, Journal of Computational Physics 140, 66-70 (1998).

[18] I. D. Boyd, "Predicting Breakdown of the Continuum Equations Under Rarefied Flow Conditions," in 23rd International Symposium on Rarefied Gas Dynamics (Whistler, Canada, 2002).

[19] I. D. Boyd, G. Chen, and G. V. Candler, Physics of Fluids 7, 210-219 (1995).

[20] T. E. Schwartzentruber, L. C. Scalabrin, and I. D. Boyd, "Hybrid Particle-Continuum Simulations of NonEquilibrium Hypersonic Blunt Body Flow Fields," in 9th AIAA/ASME Joint Thermophysics and Heat Transfer Conference (San Francisco, USA, 2006).

[21] N. G. Hadjiconstantinou, A. L. Garcia, M. Z. Bazant, and G. He, Journal of Computational Physics 187, 274-297 (2003).

[22] R. Roveda, D. B. Goldstein, and P. L. Varghese, Journal of Spacecraft and Rockets 35, 258-265 (1998).

[23] R. Roveda, D. B. Goldstein, and P. L. Varghese, Journal of Spacecraft and Rockets 37, 753-760 (2000).

[24] D. C. Wadsworth and D. A. Erwin, "One-Dimensional Hybrid Continuum/Particle Simulation Approach for Rarefied Hypersonic Flows," in 5th AIAA/ASME Joint Thermophysics and Heat Transfer Conference (Seattle, USA, 1990).

[25] D. B. Hash and H. A. Hassan, Journal of Thermophysics and Heat Transfer 10, 242-249 (1996).

[26] A. L. Garcia, J. B. Bell, and W. Y. Crutchfield, Journal of Computational Physics 154, 134-155 (1999).

[27] H. S. Wijesinghe, R. D. Hornung, A. L. Garcia, and N. G. Hadjiconstantinou, Journal of Fluids Engineering 126, 768-776 (2004).

[28] H. S. Wijesinghe and N. G. Hadjiconstantinou, International Journal of Multiscale Computational Engineering 2, 189-202 (2004).

[29] K. A. Stephani, "Development of a Hybrid DSMC/CFD Method for Hypersonic Boundary Layer Flow over Discrete Surface Roughness," Ph.D. thesis, The University of Texas at Austin 2012.

[30] J.-S. Wu, Y.-Y. Lian, G. C. C. P. Koomullil, and K.-C. Tseng, Journal of Computational Physics 219, 579607 (2006).

[31] T. E. Schwartzentruber, L. C. Scalabrin, and I. D. Boyd, Journal of Computational Physics 225, 1159-1174 (2007).

[32] G. Abbate, C. R. Klejin, and B. J. Thijsse, International Journal for Multiscale Computational Engineering 6, 1-12 (2008).

[33] G. A. Bird, Molecular Gas Dynamics and the Direct Simulation of Gas Flows (Oxford Science Publications, 1994).

[34] S. Chapman and T. G. Cowling, The Mathematical Theory of Non-Uniform Gases (Cambridge University Press, 1952).

[35] E. Nagnibeda and E. Kustova, Non-Equilibrium Reacting Gas Flows: Kinetic Theory of Transport and Relaxation Processes (Springer, 2009).

[36] Q. Sun, B. Iain D, and G. V. Candler, Journal of Computational Physics 194, 256-277 (2004).

[37] D. A. Lockerby, J. M. Reese, and H. Struchtrup, "Switching Criteria for Hybrid Rarefied Gas Flow Solvers," in Proceedings of the Royal Society A, Vol. 465 (2009), pp. 1581-1598. 